\title{
An Effective Method for Active Noise Control
}

\author{
K.Arun Kumar ${ }^{1}$, M. Vinod Kumar Reddy ${ }^{2}$ \\ ${ }^{1}$ CVR College of Engineering,ECE Department,Ibrahimpatnam,India \\ Email: arun.katkoori@gmail.com \\ ${ }^{2}$ CVR College of Engineering,ECE Department,Ibrahimpatnam,India \\ Email: vinodreddy.488@gmail.com
}

\begin{abstract}
By introducing an "antinoise" wave through secondary sources the active noise control (ANC) is achieved. Through an electronic system, the secondary sources are connected using a specific signal processing algorithm for the particular cancellation method. In this paper, we propose a method which is combination of both Feed-forward and Feedback active noise control systems called Hybrid active noise control (H-ANC) method. This method uses lower order filters and also reduces distortion generated by the feedback ANC. So this method is relatively more stable than feedback ANC. The Computation requirements for Hybrid ANC would be high and real-time implementations during one cycle. The algorithm generate the inverse waveform of the signal by collecting the noise information and update the filter coefficients effectively.
\end{abstract}

Keywords- Active noise canceling model, secondary path estimation, feed forward ANC technique, feedback ANC technique.

\section{INTRODUCTION}

ANC is a technique that can eliminate the noise emitted from a variety of sources that are present in our daily lives [1]. The Cancellation of noise makes use of the ambiguous of unfavorable interference. The final waveform depends on the frequency, amplitude and phase of the two waves, when the two sinusoidals are superimposed.

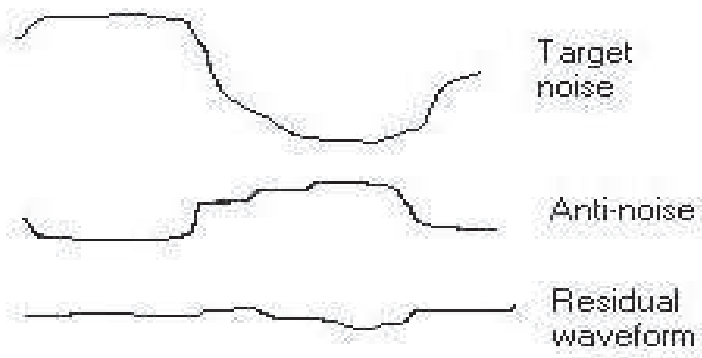

Figure 1: Physical description of active noise control (ANC)

The total cancellation will occur when original and inverse of original wave were added at a summing point at the same time [2]. In figure 1 are shown the target noise and anti-noises meeting at the junction and the residual noise waveform shown. There are two challenges--- to identify the original signal and generate the inverse without delay in all directions where these noises interact and superimpose.

Active Noise Control (ANC) is an emerging area of 'research' with a great scope for application in different fields. Most of the researchers proposed simulation studies and modeling on active noise control using MAT LAB.

For the active noise cancellation devices, there are many applications-- Cancellation of noise requires the sound or echo to be cancelled at a loud speaker, which is the source [1]. If we use headsets, the effect will work well, since that contains original sound and the canceling sound in an area near our ear. One of the applications is that, by wearing these electronic noise cancellation headsets, people who are working near aircraft or noisy factories, protect their hearing. ANC is ideal for industrial usage [3]. The engine's operation is convenient for personnel; quieting of submarines. Noise reduction eliminates vibrations that cause wear out of material and increases fuel consumption. The application of an active noise reduction produced by engines has above advantages.

\section{STRUCTURE}

Figure 2 shows headset module. The oval shape represents a microphone [5]. There are two microphones are there on the headset--one is inside the headset and the other is outside the headset.
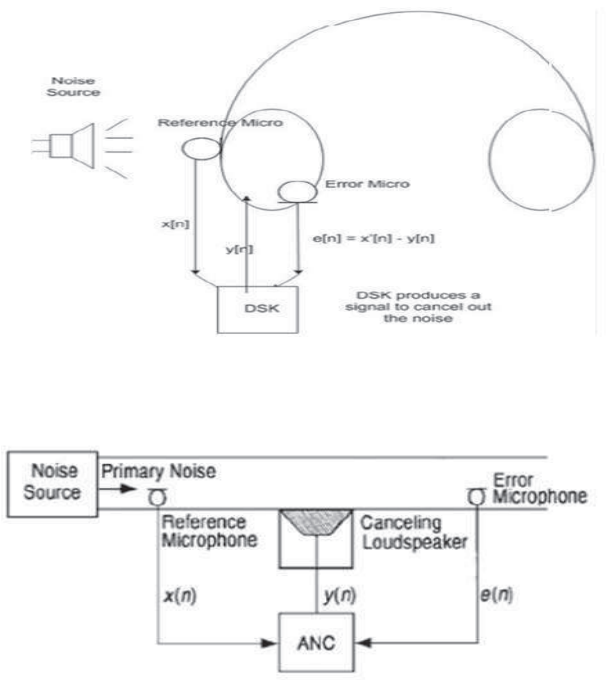

$\begin{array}{llll}\text { Figure } & \text { 2: } \\ \text { equivalent model } & \text { Structure } & \text { of } & \text { Headset }\end{array}$

The 'reference microphone' is outside microphone and is used to measure the noise near the headset. By 
processing the input and this noise, DSK tries to produce noise adaptively [6]. The 'error microphone' is inside microphone, will recognize the error between noise and from the noise source, the inverse signal is generated by the DSK. This error signal will be fed back to the DSK, which updates the filter coefficients based on the feedback.

Headset's equivalent model is shown in second structure. Because of huge amount of literature available in this structure, headset model is converting into acoustic duct model.

\section{Least Mean Square}

Computational complexity is one of the main constraints in the choice of adaptive algorithm [9]. So we have to choose an algorithm which is computationally fast for the application of Active Noise control. By taking this into consideration, Least Mean Square (LMS) algorithm became preferable choice than Recursive Least Square (RLS) [7]. by

The updated equation for the LMS algorithm is given

$$
\vec{W}(n+1)=\vec{W}(n)+\mu \cdot e(n) \cdot \vec{u}(n)
$$

Where $\mu$-step size,

$$
\mathrm{e}(\mathrm{n}) \text {-error at time ' } \mathrm{n} \text { ' and }
$$

$w^{\prime}(n)$ - the filter coefficients at time instant ' $n$ '.

$\mathrm{u}(\mathrm{n})$ - filter input vector

Replace the basic LMS shown above withe the leaky LMS,to make the system more stable.The update equation is now given by

$$
w(n+1)=b^{*} w(n)+\mu^{*} e(n) * w(n),
$$

Where $\mathrm{b}<19$

By adding the variable ' $b$ ' into the updating equation, can control the rate of change of filter coefficients $\mathrm{w}(\mathrm{n})$, over time. Also normalize the coefficients $w(n)$ to ensure that the output doesn't become large. The normalizing equation is

$$
w(n+1)=w(n+1) / \operatorname{sqrt}(\operatorname{sum}(w(n+1)))
$$

The function 'sqrt' takes a large number of cycles.

\section{Secondary Path's Off-Line Estimation}

Figure 3 shows the structure of off-line estimation of secondary path $\mathrm{S}(\mathrm{z})$ [9].By sending a sequence of training pulse to predict the secondary path $\mathrm{S}(\mathrm{z})$ before the setup of noise cancellation. There are different types of algorithms for the off-line estimation concept like FxLMS, Feedback ANC which are discussed in the following subsections.

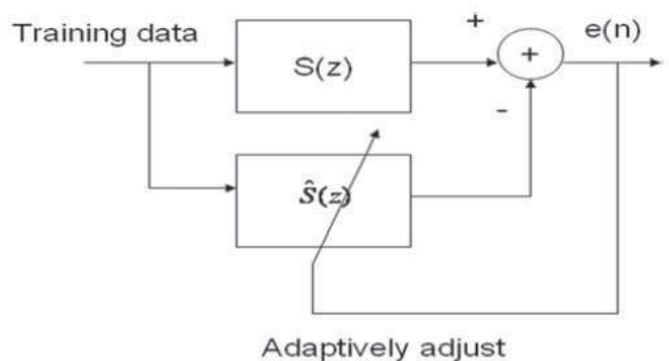

Figure 3: Structure of off-line estimation of Secondary path

\section{A) FXLMS Algorithm}

In Least Mean Square-LMS algorithm, we assume that the output of the filter $y(n)$ is perceived at the error's microphone.But this is not possible in practice, is one of the drawbacks of LMS algorithm.

If we use an identical filter in the reference signal $S^{\prime}(z)$ path and update the weight of LMS algorithm, the drawback is overcome [6]. This updated algorithm is called "Filtered-X LMS"-FxLMS algorithm shown in figure 4.
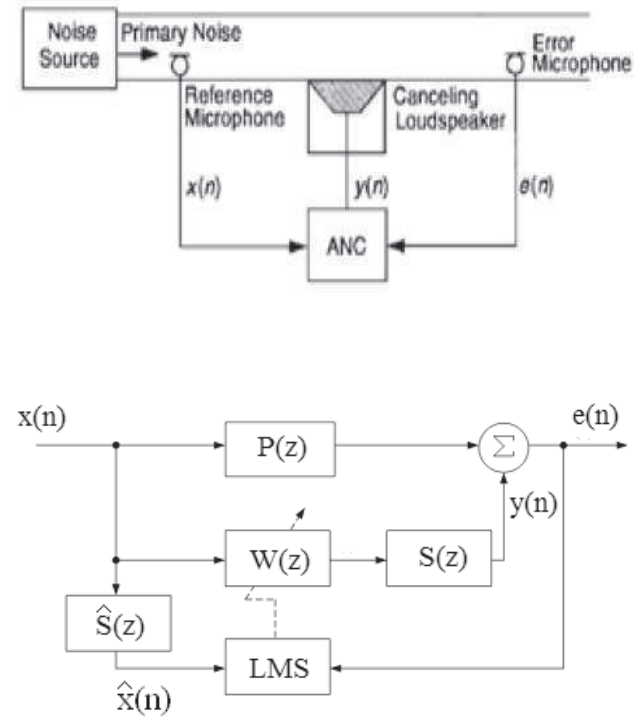

Figure 4: FXLMS ANC model

Among all other solutions, the FxLMS algorithm is the best approach. It allows the offline estimation of secondary path $\mathrm{S}(\mathrm{z})$ and very tolerant to errors made in the $\mathrm{S}^{\prime}(\mathrm{z})$ [8]. The system becomes very stable by using FIR filters to design $\mathrm{W}(\mathrm{z})$. But the convergence rate of this algorithm depends on the accuracy of secondary path estimation $\mathrm{S}(\mathrm{z})$ and if we use higher order filters, the speed of algorithm decreases [5]. The acoustic feedback presence is the major drawback of this algorithm. This is because acoustic wave from the cancelling loudspeaker to the reference microphone pairing, resulting in a depraved reference signal $x(n)$. The equations of FxLMS are 


$$
\begin{aligned}
& \text { Equations: } \\
& y(n)=w^{T}(n) x(n) \\
& \text { Updateweights: } w(n+1)=w(n)+\mu x^{\prime}(n) e(n) \\
& \text { where } x^{\prime}(n)=s(n) \otimes x(n)
\end{aligned}
$$

\section{B) Feedback ANC}

Another algorithm for offline estimation is feedback ANC. To predict primary noise and use it as a reference signal $x(n)$ is the basic idea of this algorithm. In figure 5, the error signal $e(n)$ is obtained by the subtracting the primary noise $d(n)$ from generated inverse waveform $y(n)$ [7]. During the transition, no information lost and the predicted primary noise $d(n)$ would be regenerated by adding the error signal $e(n)$ and $y(n)$. So the reference signal comes from the predicted primary noise [9]; therefore, the overall feedback mechanism performance is determined.
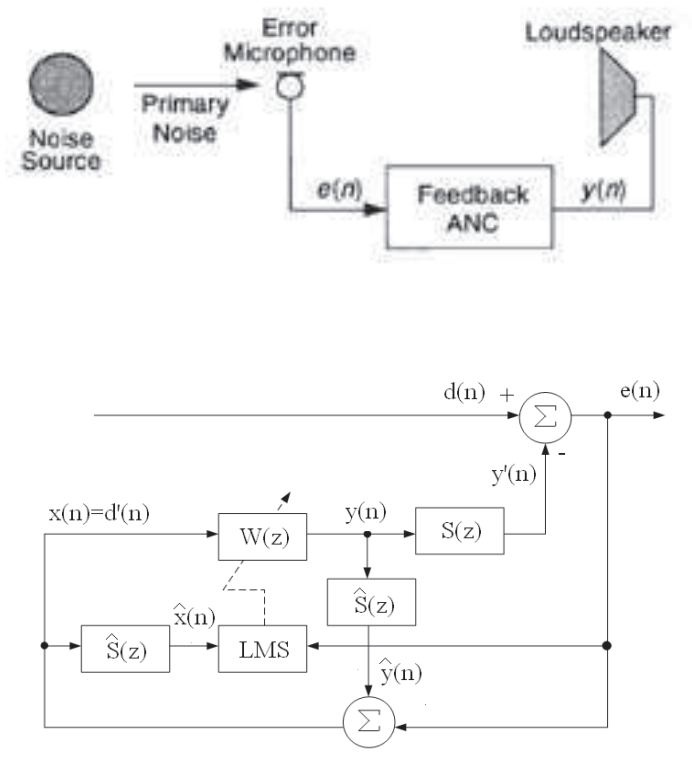

Figure 5: Feedback ANC model

By reducing the quantity of microphones from a pair to signal as well as eliminating the acoustic interference between the microphones are the two advantages of feedback ANC over basic FxLMS. The IIR structure of this algorithm produces faster convergence of LMS algorithm and the stability of the system [6]. The system is not able to catch up with aperiodic signal since half of the information for feedback system is collected from estimation in terms of frequency.

The equations of feedback ANC are

$$
\begin{aligned}
& \frac{\text { Equations: }}{y(n)=w^{T}(n) x(n)} \\
& x(n)=e(n)+\sum_{m=0}^{M-1} \hat{s}_{m} y(n-m) \\
& \text { Update weights }: w(n+1)=w(n)-\mu x^{\prime}(n) e(n)
\end{aligned}
$$

\section{C) Proposed Method-Hybrid ANC (H-ANC)}

The feedforward ANC systems discussed earlier use two sensors: a reference sensor and error sensor. The error sensor monitors the performance of the ANC system and the reference sensor measures the primary noise to be canceled [4]. The feedback ANC system uses only one error sensor and cancels the predictable noise portion of primary noise. A combination of feed forward and feedback control structure is called a "hybrid ANC system", as shown in figure 6.
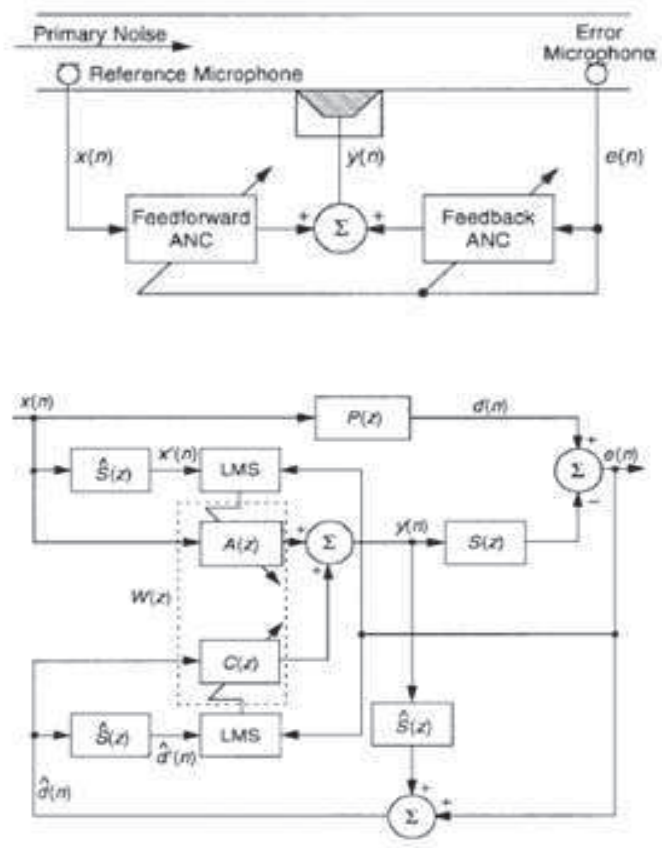

Figure 6: Hybrid ANC model

It uses lower order filters and is more stable.These are the two advantages of $\mathrm{H}-\mathrm{ANC}$, where it combines the features of feedforward and feedback systems over feedback ANC. But its computational complexity is high. The high computation requirements for Hybrid ANC would impede the real-time implementation [8]. The algorithm needs to collect the noise information, update filter coefficients and generate inverse waveform. In addition to standard LMS, protection of overflow and reset of coefficient could be necessary when the input noise is very large [5]. So the performance of the hybrid ANC is achieved.

The eqautions of proposed hybrid ANC are 


$$
\begin{aligned}
& \text { Equations: } \\
& y(n)=a^{T}(n) x(n)+c^{T}(n) \hat{d}(n) \\
& \text { Update weights : } a(n+1)=a(n)+\mu x^{\prime}(n) e(n) \\
& \qquad c(n+1)=c(n)+\mu \hat{d}^{\prime}(n) e(n)
\end{aligned}
$$

\section{Simulation Results}

The simulation of Hybrid ANC system would result in fast convergence rate by supporting the higher filter order and larger step size. And the Hybrid ANC system is sensitive to the length of filter order and less to the step size as shown in figure7. However, this observation is small comparing to the simulation result of Feedback ANC.

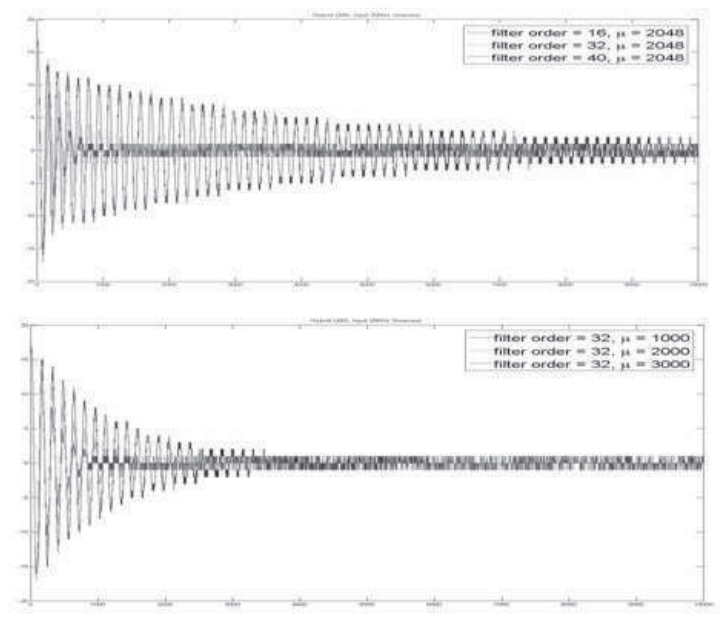

Figure 7: Hybrid ANC results

\section{Conclusions}

This paper presented a hybrid ANC system that combines the feedforward and feedback structures, updated using the FxLMS, to improve the performance of ANC in presence of acoustic feedback distortion. This fact together with the online secondary path modeling allows the system to be adjustable for any kind of secondary path change. Three different secondary path algorithms were analyzed. Computer simulations show that the hybrid ANC system provides an improved performance. Finally computer simulation results support the effectiveness of hybrid ANC system.

\section{REFERENCES}

[1] S. M. Kuo, D. R. Morgan. "Active Noise Control Systems: Algorithms and DSP Implementations" New York: Wiley Series in Telecommunications and Signal Processing Editors, 1996.
[2] B. Widrow and S. Stearns, "Adaptive signal processing," Prentice Hall, Englewood Cliffs, NJ, 1985.

[3] D. Tapia Sánchez, R. Bustamante, H. Pérez Meana, M. Nakano Miyatake, "Single Channel Active Noise Canceller Algorithm Using Discrete Cosine Transform", Journal of Signal Processing, Vol. 9, No. 2 pp. 141-151, 2005.

[4] H. Perez-Meana, M. Nakano-Miyatake, "Active Noise Canceling: Structures and

Adaptation Algorithms", Adavances in audio and Speech Signal Processing: Technologies and Applications, pag. 286- 308, Ed. Hector Pérez-Meana, Idea Group Publishing, Hershey, 2007.

[5] L. J. Eriksson, M. C. Allie, y C. D. Bremigan. "Active noise control using adaptive digital signal processing", en Proc. ICASSP, pp. 2594- 2597, 1988.

[6] S.M. Kuo, D.R. Morgan, Active noise control: a tutorial review, Proc. IEEE 87 (6) (June 1999) 943-975.

[7] S. M. Kuo and D. R. Morgan, Active Noise Control Systems - Algorithms and DSP Implementations. New York: Wiley, 1996.

[8] A. Miguez-Olivares, M. Recuero-Lopez, Development of an Active Noise Controller in the DSP Starter Kit. TI SPRA336. September 1996.

[9] S. Haykin, Adaptive Filter Theory, 2nd ed. Englewood Cliffs, NJ: Prentice-Hall, 1991. 